\title{
QUIZBOT: IMPLEMENTATION OF A CHATBOT-BASED CONVERSATIONAL INTERFACE FOR ENGAGING LEARNING CONTENTS
}

\author{
A. Ezenwoke, N. Williams \\ Covenant University (NIGERIA)
}

\begin{abstract}
In achievement environment like higher education, effective study skills are a critical factor in the pursuit of academic excellence. Arguably, most learning at a university setting happens outside the classrooms, and mainly during independent study sessions. Mobile technology can be leveraged to maximise independent study sessions in the face of increasing study contents and abounding distractions that often lead to procrastination, in order to attain optimal mastery and retention of learning contents. The advancements in chatbot technology presents an opportunity for a "personal learning assistant" that would significantly boost student's academic performance. In this paper, we report the implementation of a conversational interface for engaging of learning contents using chatbot technology. Through a conversational interface, our prototype reminds the student of their study schedules, generate multiplechoice questions from various study contents uploaded by the student, administer quizzes to help the students attain mastery of the contents.
\end{abstract}

Keywords: Chatbot, conversational interface, mobile learning, telegram, academic excellence.

\section{INTRODUCTION}

Getting a higher education diploma is one of the surest paths towards a better life; measured in terms of standard of living, quality of life, income generation, and sustenance of livelihood. Also, individuals who possess such diplomas tend to contribute better to society [1]. However, high achievements in college and completion of higher education programmes are not as easy. Some studies show that the rate of underperformance or underachievement in higher education settings is high, as is the rate of college dropouts [2]. Several attributes contribute to high academic performance such as self-efficacy, meticulosity, self-management, deploying goal-directed learning approaches etc. [3], [4]. In achievement environment like higher education, goal-directed learning approaches, which may include effective study skills, are critical factors in pursuing academic excellence. Arguably, most learning at a university setting happens outside the classrooms, and mainly during independent study sessions. Therefore, a student's ability to effectively manage their study sessions is a determinant of high academic achievement [5]-[7].

In his book, Starting Out in Community College, Dennis Congos proposed that an optimal study session includes the combination of habits that effectively manages the following: textbook reading, note-taking, memorization techniques, test preparation, concentration, and time-management [8]. Students who have imbibed these habits would be able to optimize mastery and retention of the learning contents and perform better in examinations. On the other hand, the lack of these skills leads to poor time management, stress, procrastination, low motivation, low self-confidence, and eventually, poor academic performance.

Mobile learning is the ability to learn through electronic media contents enabled by electronic devices (e.g., smartphones, tablets, and phablets) and educational, social interactions afforded by these learning platforms. Mobile learning enhances self-paced and self-regulated study in several ways as students now have more comfortable and faster access to study-related materials and general campus information [9]-[11]. Ubiquity and proliferation of internet-ready and internet-active mobile devices have enabled access to these contents. Yet, this learning mode also encourages counterproductive behaviours that contribute to poor time management and study sessions [12]-[14]. These behaviours include binge social media involvements, online games, binge chatting, and online video streaming. Noting the amount of academic work to be done within a limited time frame, any assistance (electronic or otherwise) to maximize study sessions would contribute towards a better academic outcome. We believe that mobile devices proliferation and the ubiquity of the internet, rather than constitute a vice, could be leveraged to maximize independent study sessions. 
Several study platforms that leverage mobile devices and internet already exist, such as StudyBlue and GoConqr. However, these platforms rely on the traditional GUI for users' interaction. StudyBlue ${ }^{1}$ is an online studying website for high school and college students. Users can upload and share class materials, create electronic flashcards, generate quizzes to attain and track performance. GoConqr ${ }^{2}$ is a social learning platform that provides users access to creating and using mind maps, flashcards, quizzes, and study planner. Users' learning performances can be tracked, viewed, and compared with others at the end of a course.

In recent times, the rise of conversational interfaces promises a better user experience that is more akin to the human-to-human conversation [15], [16]. Conversational User Interfaces (CUI) allow users to communicate with computers in their natural language instead of the use syntax, specific commands, or user actions. CUI uses natural language processing (NLP) for computers to understand, process, and construct meaning from human language. There are two primary forms of $\mathrm{CUI}$, including, voice assistants (e.g., Apple's Siri and Amazon's Alexa), and chatbots (e.g., Telegram bot, Facebook's Messenger).

Chatbots are intelligent agent technology with web or mobile-based software interfaces and used to conduct an online conversation with users, via text or text-to-speech [17]. The deployment of Chatbots is usually in place of direct contact with a live human agent. Chatbot-based application has been successfully deployed in customer service, to generate responses using common phrases obtained from an associated library or database, e-commerce, education, government, entertainment, finance, and healthcare [16]. Designed to mimic a human conversational partner, the advancements in chatbot technology presents an opportunity for a "personal learning assistant" for college students. Just as many CEOs in the corporate world depend on one or more executive assistants to manage their heavy workloads, we posit that engaging a virtual learning assistant to manage study-related tasks would significantly boost student's academic performance.

In this paper, we report the implementation of QuizBot, a conversational interface for engaging learning contents using chatbot technology. Through a conversational interface, our prototype can remind users (students) of their study schedules, generate multiple-choice questions from various study contents uploaded by the student, administer quizzes to help the students attain mastery of the contents. In the next sections, we present the design and prototype implementation of QuizBot.

\section{QUIZBOT: DESIGN AND IMPLEMENTATION}

\subsection{System Architecture}

QuizBot is designed based on a 3-tier architectural model. The 3-tiered architecture comprises of the frontend, question generation module and the backend. Figure 1 shows the architecture for QuizBot, while we present the details of these modules subsequently.

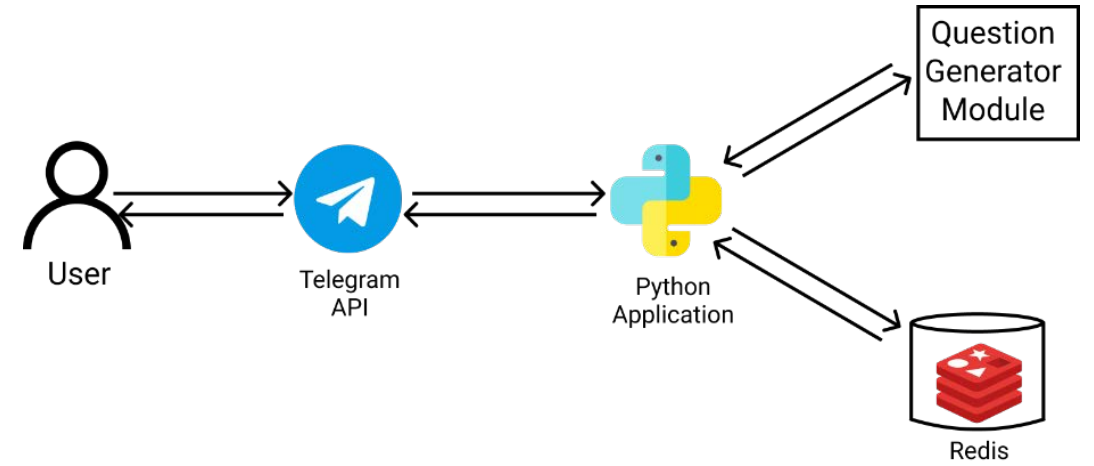

Figure 1: QuizBot Architectural Description.

Frontend: QuizBot uses the Telegram API as the platform to access the chatbot. Telegram is a cloudbased mobile and desktop messaging app focusing on security and speed; users of the application can

\footnotetext{
${ }^{1}$ https://www.studyblue.com/

${ }^{2}$ https://www.goconqr.com/
} 
send messages, exchange videos, photos, audio, stickers, and various types of files. It is available for Android, Windows, macOS, Linux and iOS users.

Backend: We built the backend with Python and Redis was. Python is an extensible, interpreted, objectoriented programming language. It supports a wide range of applications, from simple text processing scripts to interactive Web browsers. On the other hand, Redis is an open-source, in-memory data structure store, and used as a database, message broker and cache. Redis is a new generation NoSQL database [18].

Question Generator Module: We broke the process of generating questions from text files into four sub-steps, including Text extraction, Data exploration, Question generator, and Distractor generation (See Figure 2). During Text Extraction, the text from the user's learning content is extracted and fed to the system. In the Data Exploration step, we used the SQUAD 1.0 dataset to train the model. SQuAD (Stanford Question Answering Dataset) is a new reading comprehension dataset consisting of various crowd-sourced questions on a set of Wikipedia articles, where the answer to each question is a segment of text from the corresponding reading passage [19]. Keywords were identified from the uploaded textual content and used as answers to the quizzes' questions; meanwhile, QuizBot employed scikitlearn's Gaussian Naïve Bayes algorithm to classify those words. After identifying keywords in the text, the keywords are replaced with a blank space to create a fill in the gap question to which the keyword is the answer to the question; this describes the question generation step. Finally, to Generate Distractors or options, the cosine similarity and word embedding are used to identify words like the keyword.

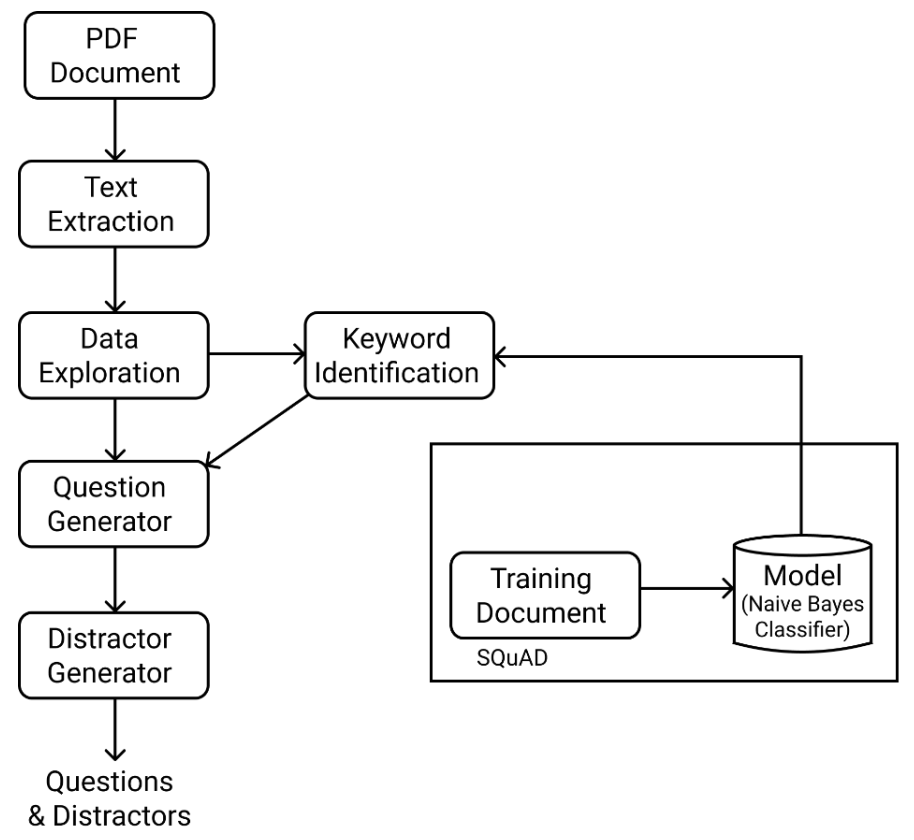

Figure 2: Process flow for the Question Generator Module.

\subsection{Modelling QuizBot Using UML}

Figure 3 and Figure 4 shows QuizBot's use case and sequence diagrams, respectively. The Use Cases in QuizBot include: Upload a file - The user provides a text-based learning content; QuizBot will generate the quizzes from the file. Choose the number of required questions - An option to choose the number of quiz items the user desires to attempt. The number of chosen questions determines the overall score. Answer questions - The chatbot generates and presents multiple choice quiz questions. The user selects their desired option, which is then reviewed by the chatbot. Show correct answers The chatbot provides feedback on the questions the users got wrong by displaying the correct answer. After that, the chatbot shows the user's total score. 


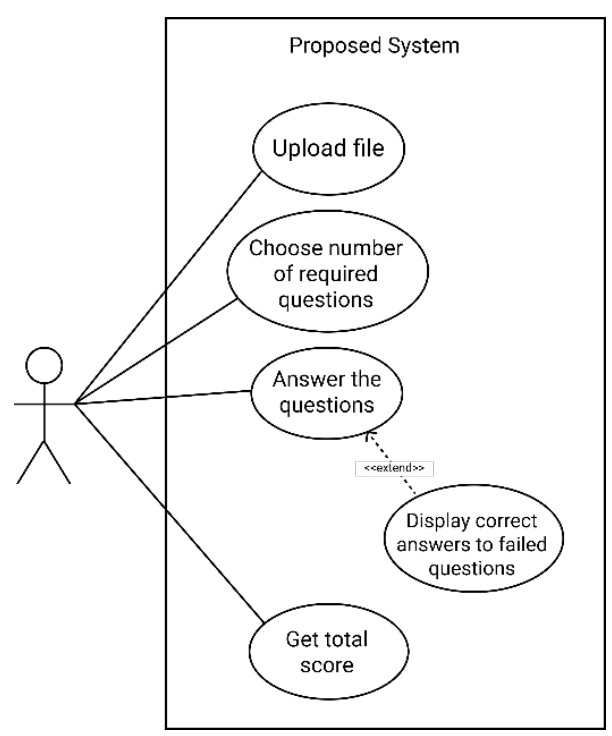

Figure 3: QuizBot Use Case Diagram.

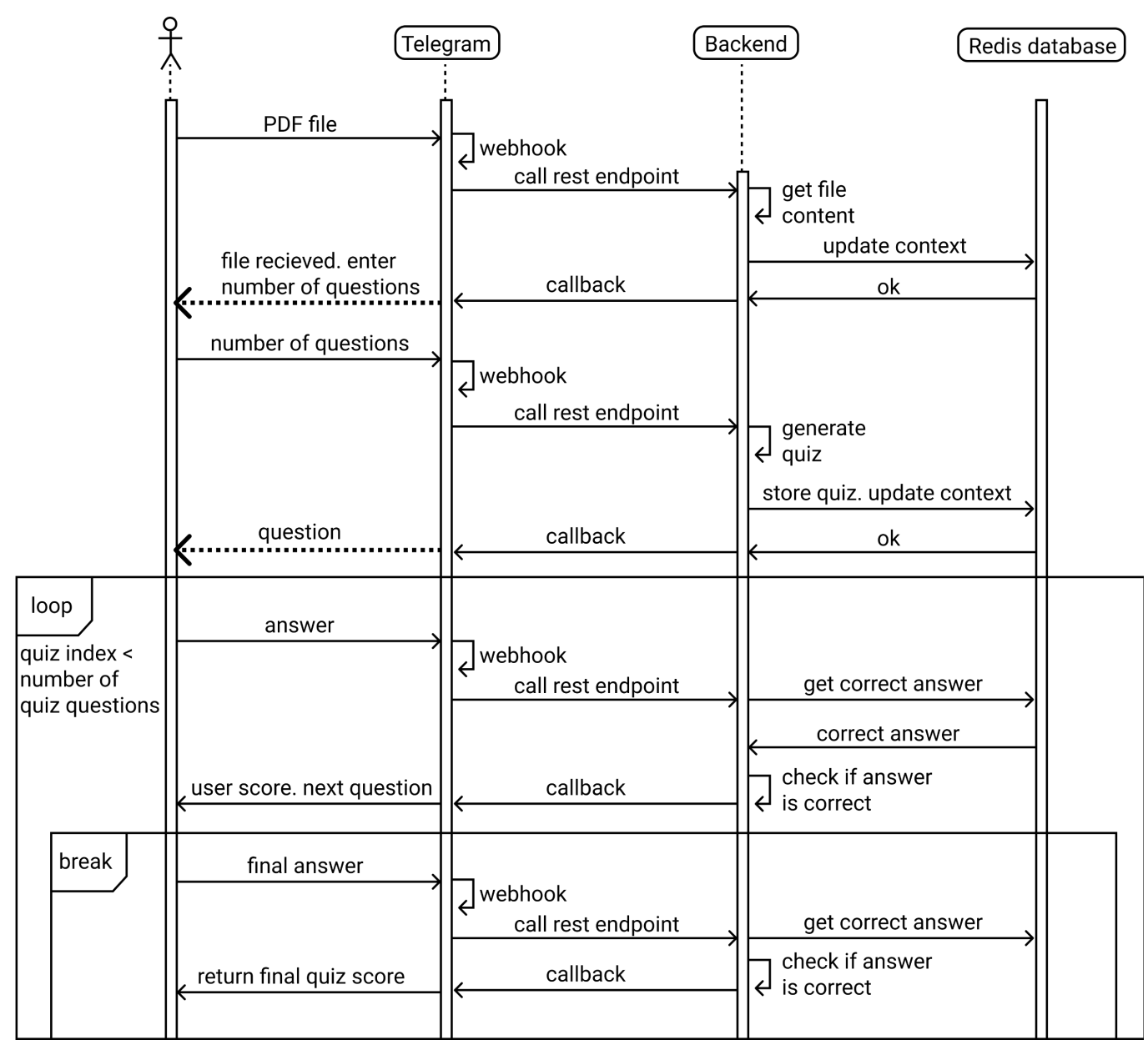

Figure 4: QuizBot Sequence Diagram.

\subsection{QuizBot Implementation}

As earlier stated, we implemented the core of QuizBot with Python programming language within flask (a lightweight micro web framework). At the same time, the Telegram bot API served as the host for the Question Generator Module and was responsible for presenting the questions and responses in a conversational interface to the user. Redis was used as a cache to store data such as the keywords or correct answers to be correctly evaluated. Visual Studio served as the QuizBot's implementation 
environment. Next, we demonstrate the utility of QuizBot in the following modules and screenshots. Figure 5 shows the welcome screen when a user launches QuizBot. The user initiates the conversation with the command "/start", and QuizBot responds with the text, "Welcome to QuizBot. I can help you generate quizzes from documents..." After the user uploads a document (in this case, the title of the document is LLL.pdf), QuizBot asks the users to specify the desired number of questions.

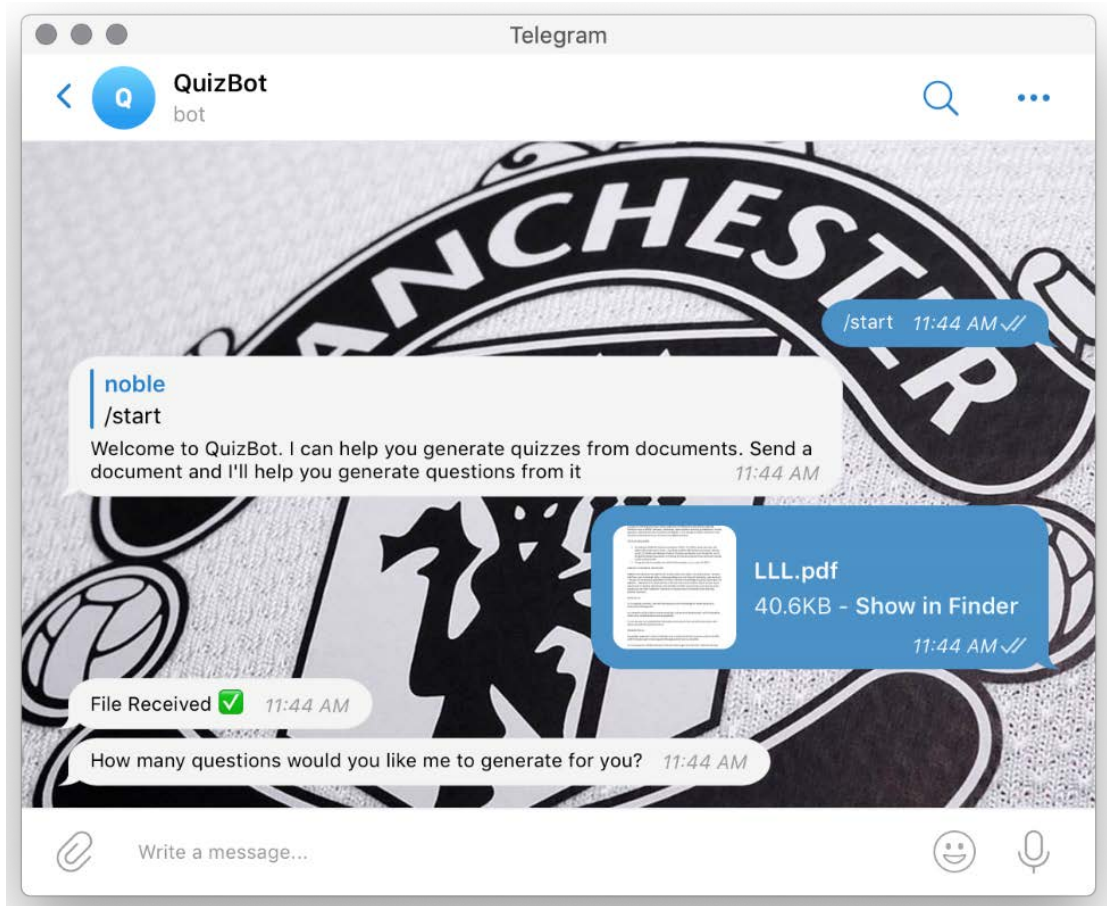

Figure 5: Welcome module.

In the screenshot shown in Figure 6, the user replied 5, as the number of questions desired. Having extracted the content of the documents, QuizBot generates five multiple-choice questions as requested by the user. The user supplies their answer, and QuizBot evaluates the answer's correctness (see Figure 7).

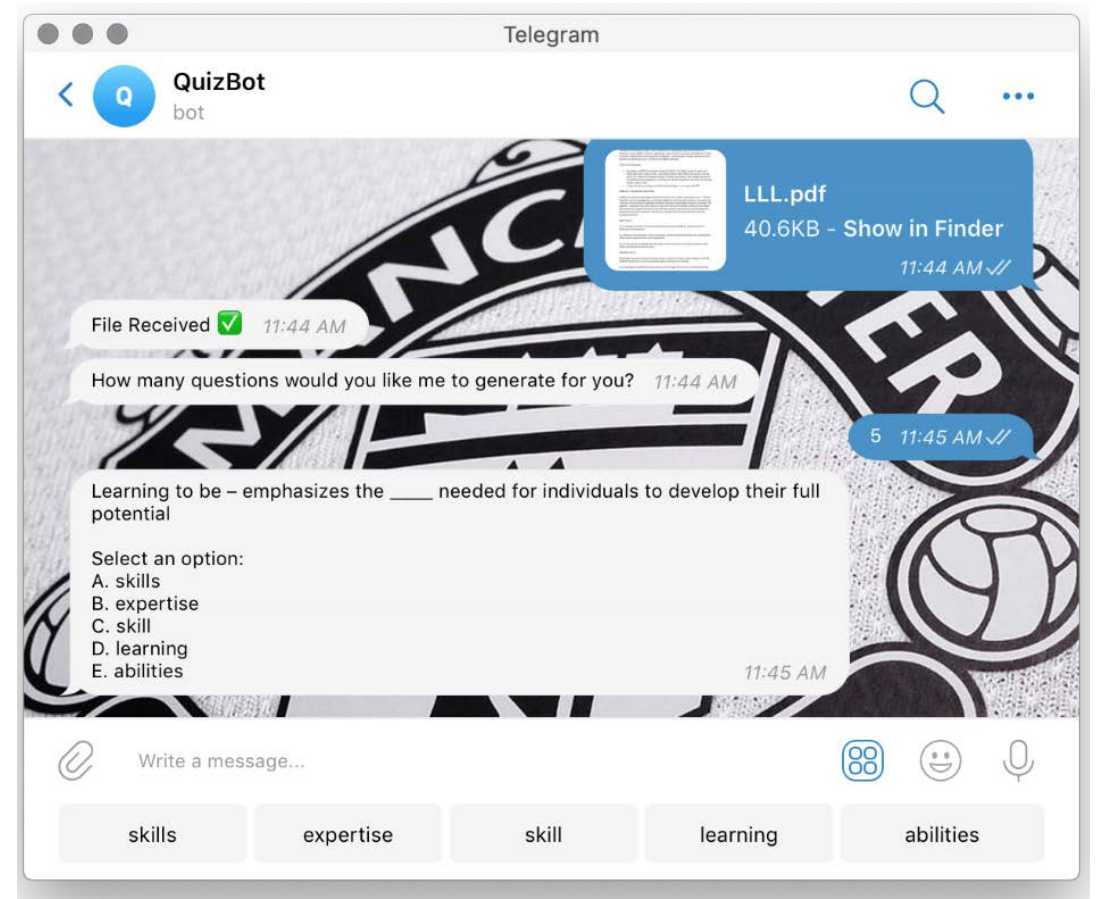

Figure 6: Question Generation module. 
In the case of a wrong answer, QuizBot provides feedback on the correct answer so the user can take note. At the end of the quiz, QuizBot notifies the user of the summary of performance, showing how much of the user's answers are correct, as shown in Figure 8.

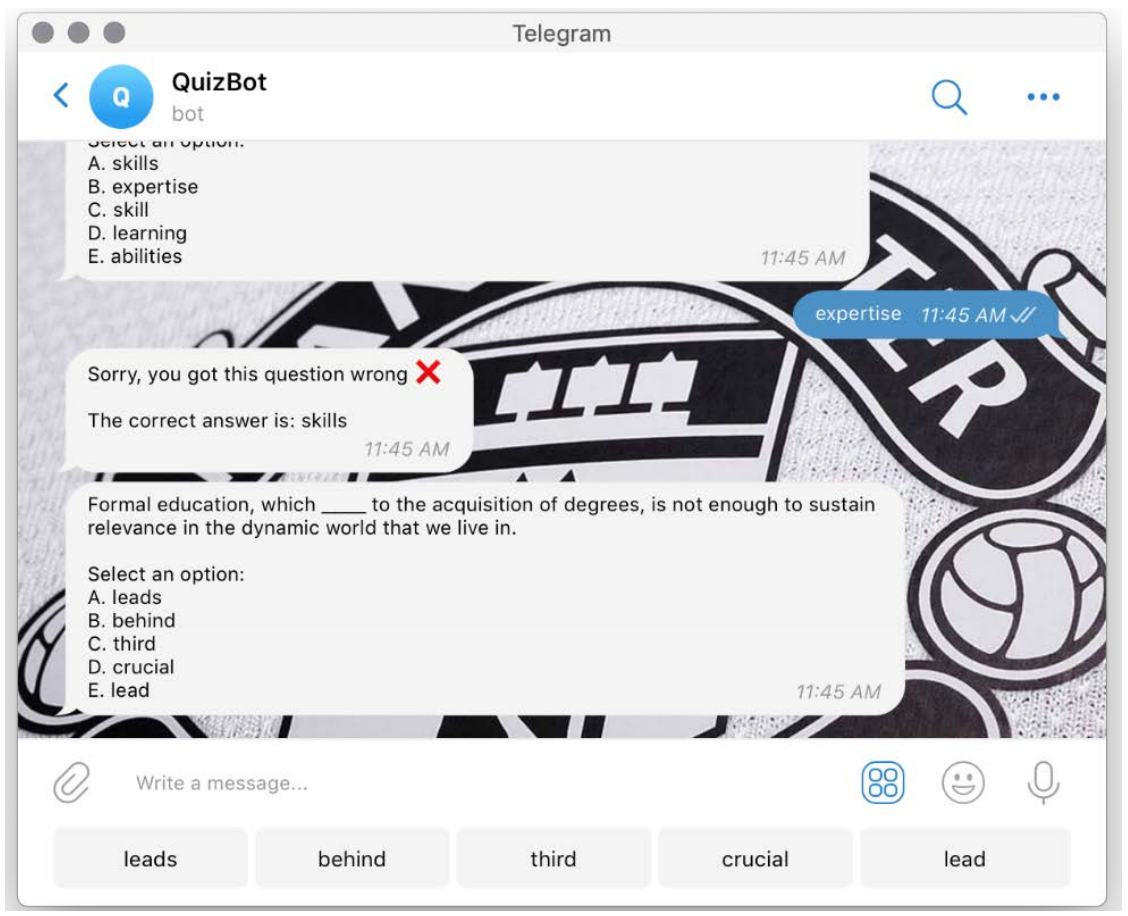

Figure 7: Wrong Selection module.

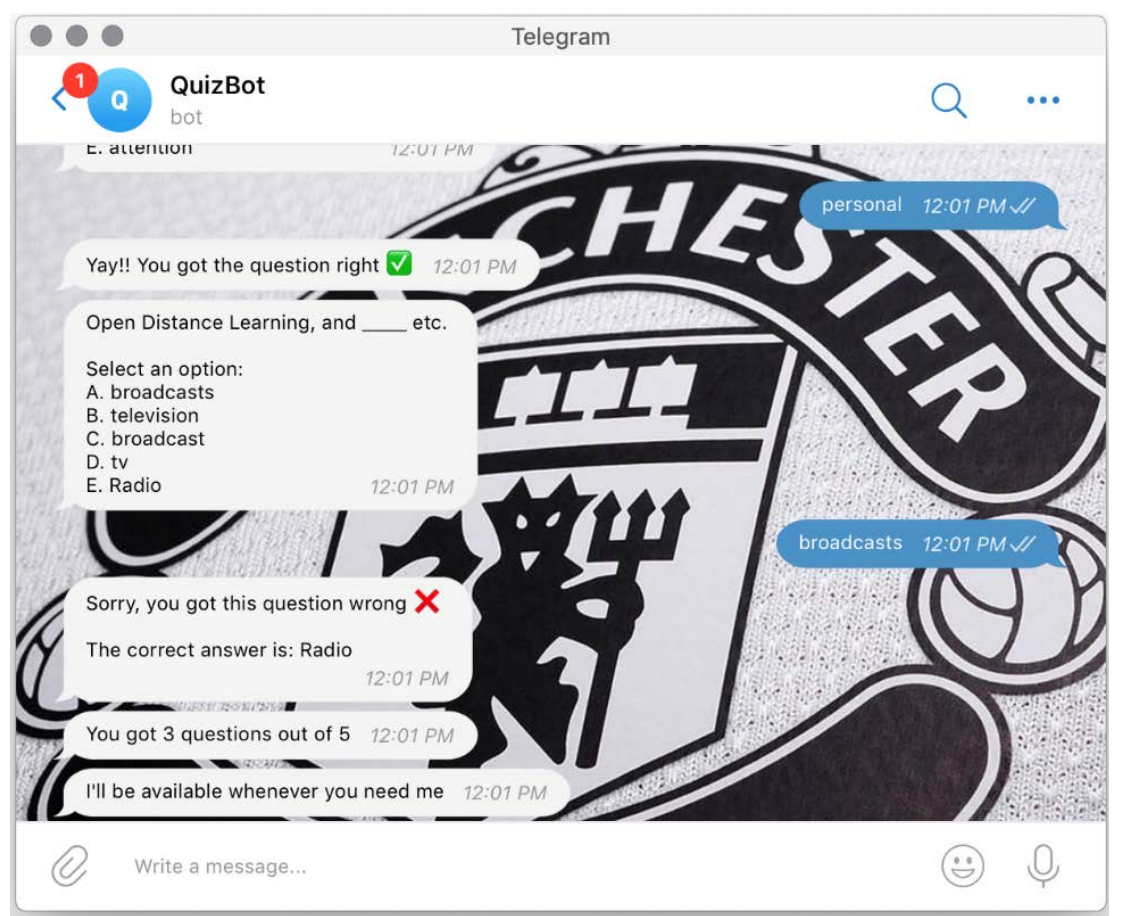

Figure 8: Final Score module.

\section{DISCUSSION}

To curb the distractions poses by mobile technology, intelligent conversational interfaces portend several benefits over traditional Ul's. First, conversational interfaces offer better simplicity in navigating the learning system over traditional UI. As demonstrated in QuizBot, the user only needs to respond to a guided conversation on the system to achieve the system's primary objective- mastery of the learning 
material. Secondly, conversational interfaces afford increased user engagement that is void of distractions. For example, QuizBot wholly immerses its users in the conversations, without the additional cognitive burden of processing graphical icons, symbols, and other information streams prevalent in traditional UI. Thirdly, conversational interfaces optimize the learners time and attention by directing the user's action and activities in line with the learning objective. QuizBot delivers curated promptings to elicit the user's responses in a time-efficient learning environment. Lastly, today's learners are mostly digital natives attuned to using chat-based technology for social and recreational purposes. Therefore, QuizBot provides a familiar learning environment for users to leverage use chat for effectively study session rather than procrastinate important study sessions. The use of QuizBot feels more like having a conversation with a study partner, personal tutor or assistant, thereby making the study session more enjoyable and rewarding.

While noting the numerous benefits and positive potentials of using conversational interfaces in learning, our current prototypical implementation has a large room for improvements. For example, this current version of QuizBot is only able to generate multiple-choice quizzes automatically. In the future, we hope to develop its capability to include essay, true/false, matching, file upload, etc. furthermore, the prototype described in this paper can only accept pdf files, while we hope to extend the acceptable formats to include documents created as .docx, .rtf, .pptx, and. xps. Finally, our current prototype uses the Telegram platform; meanwhile, we would also explore other chat and messaging apps and platform like Facebook Messenger, WhatsApp, and Skype to make QuizBot more accessible to a broader audience.

\section{CONCLUSION}

Effective study skills are a critical factor in the pursuit of academic excellence, and high academic performance is a function of making the most independent study sessions. Albeit the challenges of deploying mobile technology in enhancing learning, conversational interfaces can mitigate some of the complications and distractions afforded using mobile technology. In this paper, we introduced a QuizBot as a kind of "personal learning assistant" in a prototypical implementation of a conversational interface for engaging learning contents using chatbot technology. Based on a Telegram chat API, we demonstrated the use case of QuizBot in generating and administering a multiple-choice quiz based on the number of questions specified by the user. Our prototype will handle more complex use cases and operational over more chat-based platforms in future developments.

\section{ACKNOWLEDGEMENTS}

The authors acknowledge the Centre for Research Innovation and Discovery (CUCRID) of Covenant University for providing the required funding.

\section{REFERENCES}

[1] J. R. Abel and R. Deitz, "Do the Benefits of College Still Outweigh the Costs?," Current issues in Economics and Finance, vol. 20, 2014.

[2] I. Sandoval-Palis, D. Naranjo, J. Vidal, and R. Gilar-Corbi, "Early dropout prediction model: A case study of university leveling course students," Sustainability (Switzerland), vol. 12, no. 22, pp. 1-17, 2020, doi: $10.3390 /$ su12229314

[3] M. Schneider and F. Preckel, "Variables associated with achievement in higher education: A systematic review of meta-analyses," Psychological Bulletin, vol. 143, no. 6, pp. 565-600, 2017, doi: 10.1037/bul0000098

[4] A. Ezenwoke, O. Aigbefo, A. Sarkis, and J. Odukoya, "A preference-based grade recommender towards the attainment of a target grade point average (GPA)," Journal of Theoretical and Applied Information Technology, vol. 96, no. 13, pp. 4170-4179, 2018.

[5] M. Gettinger and J. K. Seibert, "Contributions of study skills to academic competence," School Psychology Review, vol. 31, no. 3, pp. 350-365, 2002, doi: 10.1080/02796015.2002.12086160

[6] C. Meneghetti, R. De Beni, and C. Cornoldi, "Strategic knowledge and consistency in students with good and poor study skills," European Journal of Cognitive Psychology, vol. 19, no. 4-5, pp. 628649, 2007, doi: 10.1080/09541440701325990 
[7] P. G. Nuthana and G. V. Yenagi, "Influence of study habits, self-concept on academic achievement of boys and girls *," Karnataka J. Agric. Sci., vol. 22, no. 5, pp. 1135-1138, 2009.

[8] D. H. Congos, Starting Out in Community College. McGraw-Hill Company, 2011.

[9] L. Sha, C. K. Looi, W. Chen, and B. H. Zhang, "Understanding mobile learning from the perspective of self-regulated learning," Journal of Computer Assisted Learning, vol. 28, no. 4, pp. 366-378, 2012, doi: 10.1111/j.1365-2729.2011.00461.x

[10] Y. T. Sung, K. E. Chang, and T. C. Liu, "The effects of integrating mobile devices with teaching and learning on students' learning performance: A meta-analysis and research synthesis," Computers and Education, vol. 94, pp. 252-275, 2016, doi: 10.1016/j.compedu.2015.11.008

[11] A. Mosunmola, A. Mayowa, S. Okuboyejo, and C. Adeniji, "Adoption and use of mobile learning in higher education: The UTAUT model," ACM International Conference Proceeding Series, pp. 20 25, 2018, doi: 10.1145/3183586.3183595

[12] A. A. Khan, A. Khalid, and R. Iqbal, "Revealing the relationship between smartphone addiction and academic performance of students: Evidences from higher educational institutes of Pakistan," Pakistan Administrative Review, vol. 3, no. 2, pp. 74-83, 2019, [Online]. Available: https://nbnresolving.org/urn:nbn:de:0168-ssoar-63203-4

[13] S. Qaisar, N. Akhter, A. Masood, and S. Rashid, "Problematic Mobile Phone Use, Academic Procrastination and Academic Performance of College Students," The Journal of Educational Research, vol. 20, no. 2, p. 201, 2017.

[14] S. N. Foen, N. S. I. C. Hassan, N. H. M. Nor, and N. A. A. MalekNur, "The Relationship Between Smartphone Use and Academic Performance: A Case of Students in a Malaysian Tertiary Institution," Malaysian Online Journal of Educational Technology, vol. 5, no. 4, pp. 58-70, 2013, [Online]. Available: www.mojet.net

[15] I. Celino and G. Re Calegari, "Submitting surveys via a conversational interface: An evaluation of user acceptance and approach effectiveness," International Journal of Human Computer Studies, vol. 139, 2020, doi: 10.1016/j.ijhcs.2020.102410

[16] Chatbots Magazine, "Chatbot Report 2019: Global Trends and Analysis," Chatbots Magazine, 2019.

[17] M. Shumanov and L. Johnson, "Making conversations with chatbots more personalized," Computers in Human Behavior, 2020, doi: 10.1016/j.chb.2020.106627

[18] A. K. Zaki and M. Indiramma, "A novel redis security extension for NoSQL database using authentication and encryption," 2015, doi: 10.1109/ICECCT.2015.7226101

[19] R. Pranav, Z. Jian, L. Konstantin, and L. Percy, "SQuAD: 100,000+ Questions for Machine Comprehension of Text," 2016 Conference on Empirical Methods in Natural Language Processing (EMNLP), 2016. 